\title{
Paternalismus und Placebos: Die Herausforderung der ethischen Aufklärung in der Psychotherapie
}

\author{
Charlotte Blease $^{a, b} \quad$ Manuel Trachsel ${ }^{c, d}$ Martin grosse Holtforth ${ }^{e, f}$

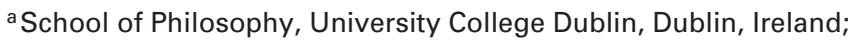 \\ ${ }^{\mathrm{b}}$ Research Affiliate, Program in Placebo Studies, Harvard Medical School, Boston, MA, USA; \\ ' Institut für biomedizinische Ethik, Fakultät für Medizin, Universität Zürich, Zürich, Schweiz; \\ dPsychiatrische Klinik Münsingen, Münsingen, Schweiz; \\ e Institut für Psychologie, Universität Bern, Bern, Schweiz; \\ ${ }^{f}$ Abteilung für Psychosomatische Medizin, Inselspital, Universitätsspital Bern, Bern, Schweiz
}

\section{Schlüsselwörter}

Psychotherapie - Ethik · Placebo · Paternalismus · Autonomie . Einverständniserklärung

\section{Zusammenfassung}

Gesundheitsfachpersonen sind heutzutage sowohl aus rechtlicher als auch ethischer Sicht dazu verpflichtet, für jede medizinische Intervention die informierte Einwilligung ihrer Patienten einzuholen. Dementsprechend hat paternalistisches Vorgehen gegenüber Patienten in den meisten ethischen Richtlinien keinen Platz mehr. Was genau bedeutet aber informierte Einwilligung im Kontext der Psychotherapie? In Bezug auf die ethische Rechtfertigung von Psychotherapie könnte z.B. die Behauptung, dass Psychotherapie nichts anderes als Placebo sei, für die informierte Einwilligung zum Problem werden. Tatsächlich wurde seit der Entstehung der modernen Psychotherapie immer wieder kritisiert, sie sei Augenwischerei und ihre Wirkung gründe auf einem Placeboeffekt. Wir argumentieren, dass im gesamten Feld der Biomedizin nach wie vor konzeptuelle Unschärfen hinsichtlich der Begriffe "Placebo" und "Placeboeffekt» bestehen. Wir sind zudem überzeugt, dass der Begriff "Placebo» im Bereich der Psychotherapie mehr Fragen aufwirft als die Auseinandersetzung damit zu beantworten vermag. Nichtsdestotrotz sind wir sicher, dass die moralisch geführte Kerndebatte über Placebo im klinischen Kontext wichtige Themen berührt, die in den psychotherapeutischen Kontext überführt werden können, nämlich: Informieren Therapeuten ihre Patienten in adäquater Weise über die Wirkmechanismen von Psychotherapie? Legen sie die potenziellen Risiken unerwünschter Nebeneffekte offen? In Anbetracht der kontinuierlichen empirischen Psychotherapieforschung folgern wir, dass Therapeuten ihren Patienten die allgemeinen Wirkfaktoren der Psychotherapie, die maßgeblich den Veränderungsprozessen während der Behandlung unterliegen, nicht hinreichend transparent machen. Somit scheint uns, dass es in der psychotherapeutischen Praxis oft zu verstecktem und unangebrachtem Paternalismus kommt. Wir sind davon überzeugt, dass sich Paternalismus in der Psychotherapie auch beim Vorliegen guter Absichten nicht rechtfertigen lässt und dass eine adäquate Offenlegung der angenommenen Wirkfaktoren für den therapeutischen Prozess nützlich ist.

(C) 2016 S. Karger GmbH, Freiburg

Das englische Original ist online abrufbar unter www.karger.com/doi/10.1159/000442928

\section{Keywords}

Psychotherapy · Ethics · Placebo · Paternalism · Autonomy · Informed consent

\section{Summary}

From a legal as well as ethical point of view, healthcare professionals are nowadays obliged to obtain informed consent of patients. Consequently, paternalism is eschewed in most ethical codes of practice. But what should informed consent mean in psychotherapy? With respect to this question, the claim that psychotherapy may be a placebo may raise grave concerns for its ethical practice. Indeed, almost since the inception of psychotherapy some scholars have claimed that psychotherapy is a sham and/or it may work as a placebo. However, we argue that in clinical biomedicine there is still much conceptual confusion about the terms 'placebo' and 'placebo effect'; moreover, we contend that the term 'placebo', when applied to psychotherapy, may invite more questions than it can easily resolve. Nonetheless, we assert that the core moral debate about clinical placebos raises important themes that are transferable to a psychotherapy context: namely: are therapists providing adequate information to patients about how psychotherapy works, and are they communicating potential risks of unwanted effects? In light of ongoing empirical research into psychotherapy we argue that therapists may be failing to mention key features (so-called common factors) that are relevant to the process of therapeutic change. We assert that current psychotherapy practice appears to exhibit misplaced paternalism in failing to provide patients with this information. We conclude that any justification for paternalism on the grounds of beneficence is unfounded and that adequate disclosure policies are likely to enhance rather than undermine the therapeutic process.

\section{KARGER} (c) 2016 S. Karger GmbH, Freiburg
Charlotte Blease, $\mathrm{PhD}$ School of Philosophy University College Dublin Dublin 4, Ireland charlotte.blease@ucd.ie 


\section{Einführung}

Die Geschichte der Medizin war bis ins späte 20. Jahrhundert eine Geschichte des ärztlichen Paternalismus. Ärzte waren dabei nicht nur Hüter des medizinischen Wissens, sondern entschieden gleichzeitig auch, wie dieses Wissen am besten eingesetzt wird und wie - falls überhaupt - Patienten über dieses Wissen in Kenntnis zu setzen sind. Gleichzeitig wurde von Patienten erwartet, die medizinische Autorität zu achten und Anweisungen entsprechend zu befolgen. Dieser Paternalismus wurde implizit damit gerechtfertigt, dass der «Doktor es schon richten wird» und dass ein «guter Patient» den Anweisungen des Doktors Folge zu leisten hat. Erst ab den später 1950er-Jahren entstand diesbezüglich in der Medizin ein kritisches Bewusstsein, und in der Folge wurden die Rechte von Patienten zunehmend ernst genommen: Patienten müssen über die vorliegende Diagnose und die verfügbaren Behandlungsoptionen sowie über deren Vor- und Nachteile wahrheitsgemäß informiert werden. In einem wegweisenden Urteil des US-amerikanischen Berufungsgerichts wurden Ärzte dazu verpflichtet, ihre Patienten über medizinische Belange so zu informieren, dass diese die Informationen auch verstehen. Dies hatte zur Folge, dass Mediziner und Ärzte heute dazu verpflichtet sind, mit ihren Patienten offen und aufrichtig umzugehen, sprich, ihre Selbstbestimmung/Autonomie $\mathrm{zu}$ respektieren. Als weiterreichende Konsequenz wird von Gesundheitsfachpersonen nun erwartet, dass sie Patienten mit angemessenen Informationen über ihre Krankheit und die verfügbaren Behandlungsoptionen versorgen, sodass diese selbst angemessene Entscheidungen über ihre Behandlung treffen können. Auch wenn immer noch nicht unbestritten ist, dass die Medizin tatsächlich frei von paternalistischen Tendenzen und Verhaltensweisen ist [Veatch, 2009; Topol, 2015], sind die ethischen Normen der Wahrhaftigkeit und des Respekts vor der Autonomie des Patienten als professionelle Haltungen in den Verhaltenskodizes von medizinischen und gesundheitsbezogenen Berufen etabliert.

In diesem Artikel werden wir auf eine bisher relativ wenig beachtete Frage eingehen [Blease, 2015a]: Ist die aktuelle psychotherapeutische Praxis paternalistisch? Wie die Medizin haben auch die klinische Psychologie und Psychotherapie den Anspruch, nicht paternalistisch zu sein. Wir gehen aber davon aus, dass es begründete Zweifel daran gibt, dass Psychotherapiepatienten genügend autonom entscheiden können. Zusätzlich nehmen wir an, dass Psychotherapieeffekte sich verbessern, wenn die ethischen Standards der informierten Einwilligung (informed consent) eingehalten werden.

An dieser Stelle möchten wir mit einer Definition von Paternalismus im therapeutischen Kontext beginnen, wobei wir zeigen, dass diese Haltung von psychologischen und psychotherapeutischen Fachverbänden explizit abgelehnt wird. Die daraus abgeleitete Notwendigkeit einer informierten Einwilligung wird vor dem Hintergrund der Beziehung zwischen Psychotherapie und Placebo kritisch durchleuchtet. Hierbei steht weniger die (unberechtigte) Gleichsetzung von Psychotherapie mit Placebo im Raum als vielmehr die Betrachtung der Probleme, die aus dem Vorhandensein der oftmals bedeutsamen Placeboeffekte für die Aufklärung und in- formierte Einwilligung von Patienten an sich entstehen. Dabei stehen Fragen nach dem «Wie weit?» und «Wann ist viel zu viel?» im Zentrum. Für die Psychotherapie stellt sich zudem das Problem, dass die sogenannten unspezifischen Wirkfaktoren wie beispielsweise die therapeutische Beziehung, Therapeuteneffekte und Patientenerwartungen - ungeachtet der erheblichen Kontroverse über deren Ausmaß und Inhalt - einen wesentlichen Anteil an den beobachteten Psychotherapieeffekten haben. Aus unserer Sicht stellt die Aufklärung und informierte Einwilligung von Patienten hinsichtlich dieser unspezifischen Wirkfaktoren das eigentliche ethische Problem in der Psychotherapie dar. Wir vertreten im vorliegenden Artikel dezidiert die Haltung, dass eine angemessene und offene Information über diese Wirkfaktoren Voraussetzung für einen respektvollen Umgang mit der Autonomie von Patienten ist und die Therapieeffekte nicht verschlechtert, sondern vielmehr die therapeutische Beziehung (und damit die Psychotherapieeffekte) verbessert [Blease, 2015a,b; Gaab et al., 2015; Trachsel et al., 2015].

\section{Paternalismus}

Aus historischer Perspektive war der Respekt vor der medizinischen Autorität eine unabdingbare Voraussetzung für die Ausübung dieser Profession, sodass - unter dem Vorsatz, ihre Patienten vor Unbill zu schützen - Patienten bis Mitte des 20. Jahrhunderts vor den negativen Auswirkungen von zu viel Wissen über die eigene Erkrankung bewahrt wurden. Wie verhält es sich aber heute mit dem moralischen Imperativ des Respekts vor der Autonomie von Patienten unter dem immer noch vorherrschenden Primat der Benefizienz/Fürsorge als Nonplusultra der Medizin?

Paternalismus kann als die Beeinflussung einer Person gegen deren erklärten Willen verstanden werden, wobei diese Beeinflussung dadurch begründet wird, dass es der Person damit entweder besser geht oder ein Schaden abgewendet werden könnte [Dworkin, 2010]. Nach dieser Definition beinhaltet paternalistisches Handeln immer auch ein bestimmtes Ausmaß an Einschränkung der Autonomie [Trachsel et al., 2013]. Paternalismus kann zudem entweder als schwacher oder starker Paternalismus beschrieben werden [Engelhardt, 1989]. Im Sinne des schwachen Paternalismus kann eine Person nur dann berechtigterweise davor bewahrt werden, sich selbst zu schaden, wenn ihre beabsichtigte Handlung zu einem substanziellen Teil unfreiwillig ist oder wenn zumindest keine Hinweise für das Gegenteil vorliegen [Feinberg, 1971]. Von starkem Paternalismus spricht man hingegen, wenn eine Person gegen ihren Willen an absolut freiwilligen Handlungen und Entscheidungen gehindert wird, die Schäden für diese Person verursachen könnten [Feinberg, 1971]. In beiden Fällen ist die Begründung paternalistischen Verhaltens üblicherweise die Verhinderung von Schaden und/oder die Sicherstellung von (klinischem) Nutzen für die Person, deren Autonomie eingeschränkt oder missachtet wird [Trachsel et al., 2013]. Da damit jede Form des Paternalismus eine Verletzung des moralischen Prinzips des Respekts vor der Autonomie beinhaltet, müssen entsprechende Handlungen mit guten Gründen gerechtfertigt werden können [z.B. Silber, 2011]. 
Es sollte an dieser Stelle darauf hingewiesen werden, dass der Respekt vor der Autonomie und das Streben nach Benefizienz/Fürsorge nicht unbedingt in einem moralischen Konflikt zueinander stehen müssen. Wenn es jedoch zu einer Spannung zwischen diesen beiden moralischen Prinzipien kommt, muss erkannt werden, dass das Streben nach Benefizienz den Respekt vor der Autonomie einschränkt und umgekehrt. Zusammenfassend besteht dieser moralische Konflikt darin, dass - unabhängig von der letztlich getroffenen Handlungsentscheidung - eines dieser zwei moralischen Prinzipien der biomedizinischen Ethik verletzt wird [Beauchamp und Childress, 2009].

Wie geht nun die Psychotherapie mit diesem ethischen Konflikt um? Zur Beantwortung dieser Frage sollen in einem ersten Schritt zunächst die ethischen Richtlinien der Psychotherapie-Fachverbände betrachtet werden.

\section{Umgang mit Paternalismus in der Psychotherapie}

In Deutschland ist die Ausübung der Psychotherapie gesetzgeberisch auf Fachärzte für Psychiatrie, Psychosomatik und Psychotherapie sowie psychologische Psychotherapeuten beschränkt. Die Berufsordnung der Deutschen Psychotherapeutenkammer enthält einen Artikel (\$ 7) über die Aufklärungspflicht von psychologischen Psychotherapeuten gegenüber ihren Patienten. Dabei wird verlangt, dass Patienten vor dem Beginn einer psychotherapeutischen Behandlung über folgende Aspekte angemessen informiert werden: Indikation, Art der Behandlung, Therapieplan, gegebenenfalls Behandlungsalternativen und mögliche Behandlungsrisiken, Honorarregelungen, Sitzungsdauer und Sitzungsfrequenz und die voraussichtliche Gesamtdauer der Behandlung. Ähnliche Bestimmungen werden in den ethischen Richtlinien der Deutschen Gesellschaft für Psychologie (DGP) und vom Berufsverband Deutscher Psychologinnen und Psychologen (BDP) formuliert:

«Psychologen müssen ihre Klienten / Patienten über alle wesentlichen Maßnahmen und Behandlungsabläufe unterrichten und sich ihrer Einwilligung versichern. Bei heilkundlichen Behandlungen haben sie auf ggf. bestehende Risiken und Alternativbehandlungen hinzuweisen. Die Hinweispflicht umfasst auch Fragen des Honorars und der Kostenerstattung.» (D.I.2.)

In der Schweiz beinhaltet die Berufsordnung der Föderation der Schweizer Psychologinnen und Psychologen als größtem Berufsverband folgende spezifische Regeln für die psychotherapeutische Tätigkeit:

«Mitglieder klären ihre Patientinnen und Patienten bzw. deren gesetzliche Vertretung in verständlicher und sachlicher Form hinreichend auf, insbesondere über: a) die beabsichtigten Verfahren oder Methoden und das Setting, b) allfällige mit der Behandlung verbundene Risiken und Behandlungsalternativen, c) die finanziellen Bedingungen, namentlich das Honorar oder die Vergütung durch die Grund- oder Zusatzversicherung und die Art der Verrechnung versäumter Stunden, d) die Schweigepflicht. Sie klären mit den Patientinnen und Patienten insbesondere die beabsichtigten Ziele und die voraussichtliche Dauer der Behandlung. Mitglie- der weisen darauf hin, wenn sie ihre Tätigkeit in einem delegierten Verhältnis für eine Ärztin oder einen Arzt ausüben.»

In den USA legt die American Psychological Association (APA) fest, dass «(psychologists should) [o]btain the informed consent of the individual (... and they) should seek to promote accuracy, honesty, and truthfulness in the science, teaching, and practice of psychology» (3.10, [APA, 2010]). Entsprechend ist im Ethical Framework for Good Practice der British Association for Counseling and Psychotherapy (BACP) festgehalten, dass «(practitioners should) ensure accuracy in any advertising or information given in advance of services offered; seek freely given and adequately informed consent ... The principle of autonomy opposes the manipulation of clients against their will, even for beneficial social ends» [BACP, 2013]. Darüber hinaus sind in Großbritannien und den USA Psychiater dazu angehalten, die Zustimmung des Patienten oder einer anderen befugten Instanz einzuholen [GMC, 2010], weil die Vorenthaltung medizinischer Informationen unmoralisch ist [AMA, 2006].

Es zeigt sich also, dass die oben genannten ethischen Richtlinien und Berufsordnungen in Bezug auf die Respektierung der Autonomie von Psychotherapiepatienten relativ einheitlich und normativ sind: Es wird verpflichtend erwartet, dass Psychotherapeuten ungefragt, umfassend und ehrlich über die Behandlung informieren. Dieser Respekt vor der Autonomie des Patienten wiegt stärker als das Bestreben nach Benefizienz/Fürsorge, und entsprechend lehnen diese ethischen Richtlinien implizit jeglichen Paternalismus ab. Mit anderen Worten: Um zu therapieren, braucht es zuerst die informierte Einwilligung des zu Therapierenden.

Worüber aber sollten Patienten entsprechend dieser ethischen Richtlinien also informiert werden? Die APA schreibt hierzu, dass «Psychologen ihre Patienten in Abhängigkeit des jeweiligen Behandlungsvorgehens darüber informieren sollten, welchen Ansatz sie zur Behandlung des vorliegenden Problems einsetzen und welche Techniken oder Methoden wie zum Beispiel Exposition, Traumanalysen, Familienstellungen oder diagnostische Anamnesen dieser Ansatz beinhaltet. ... Darüber hinaus sollten Psychologen ihre Patienten über alle Aspekte informieren, die diese benötigen, um eine informierte Entscheidung für oder gegen die Behandlung zu treffen» [Fisher und Oransky, 2008]. Darüber hinaus können «manche Psychotherapeuten ihre Patienten über den empirischen Kenntnisstand zur betreffenden Psychotherapieform informieren». Dieser Zusatz lässt vermuten, dass der Nachweis der Wirksamkeit einer bestimmten Behandlungsform kein notwendiger Bestandteil der informierten Einwilligung darstellt; was zählt, ist die Information über die Behandlungsform und die dabei eingesetzten Methoden. Tatsächlich kann hier sogar angenommen werden, dass dieser optionale Umgang mit der Evidenz psychotherapeutischer Behandlungen ein Abbild der Haltung ist, dass Psychotherapie evidenzbasiert sein kann, aber nicht sein muss. Aus einer wohlwollenderen Perspektive kann dies aber auch so verstanden werden, dass alle verwendeten psychotherapeutischen Behandlungen sowieso evidenzbasiert seien und es dem Psychotherapeuten freigestellt ist, Patienten darüber zu informieren oder nicht.

Entsprechend verlangt der ethische Verhaltenskodex der BACP, dass «alle Psychotherapeuten dazu angehalten sind, ihr professio- 
nelles Wissen und ihre Erfahrung zum Wohle ihrer Patienten einzusetzen [...] [und] die Weiterbildung in [...] Psychotherapie die Standards und Vorgehensweisen vermittelt, die man in der Praxis erwartet» [BACP, 2013]. Diese Bestimmungen schreiben wie die der APA vor, dass die Psychotherapeuten ihre Patienten über die betreffende psychotherapeutische Behandlung umfassend aufklären. Es gilt aber festzuhalten, dass beide Vorgaben von der Validität der betreffenden psychotherapeutischen Behandlungstheorie ausgehen, jedoch ohne diese zu spezifizieren.

Zusammenfassend ist festzuhalten, dass sich die Information und Aufklärung von Patienten in den ethischen Richtlinien auf die Methoden bezieht (oder beschränkt), die der Psychotherapeut einsetzt und in denen dieser ausgebildet wurde. Ob eine solche Information und Aufklärung dann auch tatsächlich in der Praxis entsprechend umgesetzt wird, ist unklar [siehe z.B. Dsubanko-Obermayr und Baumann, 2010]. Es ist jedoch hervorzuheben, dass es dem Psychotherapeuten freigestellt ist, den Patienten auch über die wissenschaftliche Evidenz der Behandlungsentscheidung zu informieren. Da dieser letzte Punkt in der medizinischen Interventionsforschung eng mit der Frage nach der Spezifität, verstanden als die Anteile der Interventionseffekte über den Placeboeffekt hinaus, verknüpft ist, soll die Beziehung zwischen Psychotherapie und Placebo im Folgenden näher diskutiert werden.

\section{Psychotherapie und Placebo: Eine Analyse der therapeutischen Beziehung}

Die Wirkmechanismen psychotherapeutischer Behandlungen sind ein sowohl fruchtbares als auch heftig und kontrovers diskutiertes Forschungsgebiet. Von Anfang an wurde diese Diskussion von dem Vorurteil begleitet, dass solche «Redekuren» (talking cures) pseudowissenschaftlich, Täuschung oder nichts weiter als Placebo seien [Rosenzweig, 1936; Rosenthal und Frank, 1956; Frank, 1983]. Diese Ansicht ist nicht nur aus empirischer Sicht bedeutsam - auch wenn und vor allem weil die Psychotherapie heute nachweislich eine wirksame Behandlung von psychischen Störungen darstellt [Lambert, 2013] - sondern hat auch maßgeblich Einfluss auf den ethischen Status der Psychotherapie.

Um die Behauptung «Psychotherapie ist nichts als Placebo» und deren Auswirkung auf die informierte Einwilligung angemessen beurteilen zu können, ist es in einem ersten Schritt notwendig, Placebo und dessen Effekte angemessen zu definieren. Hierbei muss aber beachtet werden, dass es bis dato keine unumstrittenen Definitionen von Placebo gibt und ein In-Bezug-Setzen zur Psychotherapie dadurch erschwert ist. Der Versuch einer Definition von Placebo wurde deswegen von Turner damit verglichen, «verschiedene unterschiedliche Bestandteile in einen Topf [zu] werfen», was dann zur Folge hat, dass «man am Ende alle verschiedenen Farben zu einem undefinierten Braun zusammenrührt» [Turner, 2012].

Placebos werden oft als «inaktive Substanz» begriffen, was aber in Anbetracht neuerer Forschungsergebnisse so nicht mehr aufrechterhalten werden kann. Hierbei werden Placeboeffekte nicht nur durch Placebos allein, sondern auch über verbale und nonver- bale sozio-emotionale Hinweisreize ausgelöst [Kaptchuk et al., 2008; Jensen et al., 2012], sodass es sinnvoller ist, Placeboeffekte als Kontexteffekte beziehungsweise als «Effekte, die aus der PatientBehandler-Beziehung entstehen» [Di Blasi und Harkness, 2001] zu verstehen. Darüber hinaus muss berücksichtigt werden, dass verschiedene Behandlungskomponenten, wie beispielsweise Markennamen, Verabreichungsmodalität und Preis, nicht nur die Effekte von Placebos, sondern auch die von Verum-Interventionen beeinflussen. Placeboeffekte können also nicht nur bei Placebos selbst, sondern bei jeglicher Intervention auftreten [Kaptchuk et al., 2000]. Wenn man nun Placeboeffekte über «Effekte, die aus der PatientBehandler-Beziehung entstehen» [Di Blasi und Harkness, 2001] definiert, dann wäre die Schlussfolgerung nachvollziehbar, Psychotherapie a priori als Placebo zu definieren [Kirsch, 2005]. Ein anderer Ansatz geht davon aus, dass Placebos unspezifische Behandlungseffekte darstellen, sodass die Spezifität einer Behandlung festlegt, ob diese nun ein Placebo ist oder nicht. Entsprechend definieren Shapiro und Shapiro [1997], dass «der Placeboeffekt primär der unspezifische psychologische oder psychophysiologische therapeutische Effekt ist, der durch das Placebo verursacht wird». Um den Placebobegriff zu entmystifizieren und um Forschung hinsichtlich relevanter Komponenten und Mechanismen zu stimulieren, haben Castonguay und grosse Holtforth [2005] vorgeschlagen, dass es sich bei Placeboeffekten um «noch nicht spezifizierte Effekte» handelt. Ein weiterer Ansatz nach Grünbaum [1981, 1986] versteht Placebos als Behandlungen, deren charakteristische Bestandteile ohne absichtliche oder unabsichtliche Täuschung keine therapeutischen Effekte auf eine vorliegende Störung oder Erkrankung haben.

Zusammenfassend sind die bisher genannten Definitionsansätze problematisch, weil sie entweder auf einem zirkulären Verständnis von Placebos und Placeboeffekten beruhen oder weil sie durch den Bezug zu einer jeweiligen Behandlungstheorie kein allgemeingültiges Verständnis erlauben [Greenwood, 1997].

Wie nun soll - unabhängig vom Problem der noch ungeklärten Definition von Placebos und ihren Effekten in der Biomedizin der Bezug zur Psychotherapie hergestellt werden? Betrachten wir dazu den Vergleich einer psychotherapeutischen Behandlung mit einer sogenannten Placebotherapie in einer randomisierten Placebo-kontrollierten Studie, beispielsweise einer «attention placebo control group». Diese sogenannte Placebotherapie wird üblicherweise eingesetzt, um die Auswirkungen der Zeit und Aufmerksamkeit, die die Teilnehmer der «echten» psychotherapeutischen Behandlung erhalten, zu kontrollieren. Das grundlegende Problem dabei ist, dass dabei das epistemologische Argument, die Kontrolltherapie so zu gestalten, dass sie bis auf das spezifische Element der $\mathrm{zu}$ untersuchenden Behandlung mit eben dieser vergleichbar beziehungsweise von dieser ununterscheidbar ist, missachtet wird [vgl. Howick, 2011; Turner, 2012]. Die Voraussetzung der Ununterscheidbarkeit ist im biomedizinischen Kontext (in der Regel) einfach und gut, in der Psychotherapie hingegen ungleich schwerer umsetzbar, da die Psychotherapie (in der Regel) eine komplexere Angelegenheit ist. Neben der Schwierigkeit, die Verum-Bestandteile sowohl konzeptionell als auch prozedural von den sogenannten «Placebo»-Bestandteilen zu trennen, ist auch die doppelblinde 
Durchführung - als Gold-Standard der biomedizinischen Interventionsforschung - unmöglich, schlicht aus dem Grund, dass Psychotherapeuten (hoffentlich) immer wissen, was sie machen.

Zusätzliche Probleme für die Psychotherapie entstehen aufgrund des Umstands, dass Placebos (und Placeboeffekte) mit mehreren Faktoren in Verbindung gebracht werden, die als allgemeine Wirkfaktoren über verschiedene Psychotherapieansätze hinweg betrachtet werden. Beispielsweise wurden die therapeutische Beziehung, das Therapeutenverhalten, die Behandlungserwartungen von Patient und Behandler sowie die Information des Patienten über mögliche Krankheitsursachen als Placebo betrachtet, da all diese Faktoren unspezifische oder noch nicht spezifizierte Behandlungskomponenten darstellen [Frank und Frank, 1993; Jopling, 2008].

Es findet sich gar die Position, dass die Anwendung des Placebokonzepts auf die Psychotherapie keinen Sinn macht:

«Der Placeboeffekt in der Medizin ist das Produkt der nichtphysischen Eigenschaften einer Behandlung. Wenn nun aber die Wirkung der Psychotherapie - nomen est omen - über die nichtphysischen Eigenschaften dieser Intervention verursacht wird, dann wären Psychotherapieeffekte ipso facto Placeboeffekte und entsprechend Psychotherapie ein Placebo» [Kirsch et al., 2015; Kirsch, 2005].

Eine solche reductio ad absurdum ist natürlich problematisch, da hier ontologische Belange («Was ist ein Placebo?») mit epistemologischen Aspekten («Wie können in Wirksamkeitsstudien unspezifische Faktoren kontrolliert werden?») vermischt werden. Und auch wenn wir anerkennen, dass die klinische und experimentelle Ethik von einer Berücksichtigung der Placeboforschung profitieren kann, sprengt eine abschließende Beurteilung des Verhältnisses zwischen Psychotherapie und Placebo den Rahmen dieser Abhandlung zum ethischen Status der Psychotherapie [Gaab et al., 2015]. Gleichwohl stellen wir fest, dass die ethische und konzeptuelle Analyse des Placebos eine wichtige gemeinsame Botschaft enthält: Die Anwendung des Placebokonzeptes impliziert immer eine Vernachlässigung oder Unterlassung der angemessenen Information und informierten Einwilligung des Patienten bezüglich zentraler therapeutischer Komponenten der Behandlung. Mit der Gleichsetzung von Psychotherapie mit Placebo werden somit fundamentale Komponenten der Behandlung grundsätzlich verzerrt wiedergegeben [Blease, 2015c]. Entsprechend konzentrieren wir uns hier auf die Möglichkeit, dass Psychotherapie Patienteninformation und informierte Einwilligung vernachlässigt oder gar unterlässt. Im Folgenden sollen deswegen zuerst die Kriterien und Ansprüche einer angemessenen Patienteninformation und informierten Einwilligung dargestellt werden, um dann den Nachweis zu erbringen, wie und/oder dass Psychotherapeuten diesen Ansprüchen gegenwärtig noch nicht gerecht werden.

\section{Kriterien und Ansprüche einer angemessenen Patienteninformation und informierten Einwilligung}

Da schon allein aufgrund zeitlicher Einschränkungen Patienten nicht über alle relevanten Aspekte einer Behandlung erschöpfend und abschließend informiert werden können, muss sich die Aufklärung des Patienten auf die wichtigsten Aspekte der Behandlung beschränken, die den Patienten dazu befähigen, der betreffenden Behandlung zuzustimmen beziehungsweise diese abzulehnen [Beauchamp und Childress, 2009, S. 117]. Die drei Grundpfeiler der Patienteninformation und informierten Einwilligung umfassen nach Beauchamp und Childress [2009]: (1) Voraussetzungen zur Erfüllung der Patienteninformation und der informierten Einwilligung, (2) Inhalt der Patienteninformation, (3) Inhalt der informierten Einwilligung.

Wie lassen sich diese drei Aspekte im Bereich der Psychotherapie umsetzen?

Betrachten wir zuerst die Voraussetzungen für die Patienteninformation und die informierte Einwilligung. Auch wenn Patienten in psychotherapeutischer Behandlung in der Regel starke psychische Störungen und Probleme und entsprechend starke Beeinträchtigungen psychischer Funktionen aufweisen, bedeutet dies nicht automatisch, dass die Voraussetzungen für eine angemessene Patienteninformation und für eine informierte Einwilligung nicht mehr gegeben sind. In Großbritannien verlangt beispielsweise der Mental Capacity Act von 2005 [UK Department for Constitutional Affairs, 2005], dass die Beweislast hierfür aufseiten des Behandlers und nicht aufseiten des Patienten liegt: «Der erste Grundsatz ist, dass behandelte Personen die Fähigkeit haben, eigenständige Entscheidungen zu treffen oder Handlungen zu begehen, außer es wird eindeutig festgestellt, dass dies nicht der Fall ist. Dies heißt, dass man nur dann im Interesse des Patienten entscheiden oder handeln kann, wenn die betreffende Person nachgewiesenermaßen die notwendige Fähigkeit dazu nicht hat oder wenn dies begründet angenommen werden kann.»

Um nun den unterschiedlichen Annahmen und praktischen Umsetzungen in verschiedenen psychotherapeutischen Ansätzen gerecht zu werden, müssen Patienten - auch wenn sie verschiedene Symptome und Beeinträchtigungen aufweisen - die Fähigkeit haben, Gespräche und Diskussionen reflektiert zu führen, regelmäßige Sitzungen zu besuchen und/oder Hausaufgaben zu erledigen. Entsprechend gehen wir deswegen davon aus, dass erwachsene Patienten in der Regel die Voraussetzungen für eine Information über die Psychotherapie und eine entsprechende informierte Einwilligung aufweisen.

In Bezug auf den Inhalt der Patienteninformation wurden bislang verschiedene Heuristiken eingesetzt. Beauchamp und Childress [2009, S. 122] beschreiben dazu drei Ansätze, die helfen können, den normativen Anforderungen gerecht zu werden:

Die Heuristik des professionellen Standards geht davon aus, dass professionelle Gewohnheiten im Praxisalltag bestimmen, über was und in welchem Umfang Patienten bezüglich ihrer Behandlung aufgeklärt werden. Damit wird angenommen, dass Angehörige des Gesundheitswesens nach eigenen und professionellen Gepflogenheiten vorgehen können. Dieses normative Verständnis war so lange Standard im Gesundheitswesen, bis es in berufsethischen Kodizes als unethisch eingestuft wurde.

Die Heuristik des vernünftigen Standards geht davon aus, dass Angehörige des Gesundheitswesens so viele Informationen über 
eine Behandlung geben sollten, wie eine hypothetisch vernünftige Person dies in einer hypothetischen Behandlungssituation bräuchte. Dieser Ansatz geht also retrospektiv von einer vernünftigen Person aus, um darüber zu spekulieren, welche Informationen ein Patient in einer bestimmten Behandlungssituation gerne erhalten würde.

Die Heuristik des subjektiven Standards bezieht hingegen individuelle Bedingungen des Patienten ein: «Patienten haben möglicherweise unkonventionelle Annahmen, außergewöhnliche Gesundheitsprobleme oder einzigartige Herkunftsfamilien, was dann auch dazu führt, dass diese Patienten andere Informationen benötigen als ein hypothetischer vernünftiger Patient» [Beauchamp und Childress, 2009, S. 123].

Wie lassen sich diese Standards beurteilen? Die Heuristik des professionellen Standards - dass Angehörige des Gesundheitswesen in der Lage sind, die Präferenzen von Patienten in Bezug auf deren Behandlungswünsche zu erkennen - ist empirisch nicht haltbar, da beispielsweise Ärzte darin nachweislich schlecht abschneiden [z.B. Street und Haidet, 2011]. Wichtiger erscheint aber der Einwand, dass «der professionelle Standard das Anrecht des Patienten auf eine autonome Entscheidung verletzt» [Beauchamp und Childress, 2009, S. 122]. Entsprechend wäre das, selbst wenn Angehörige des Gesundheitswesens die Präferenzen ihrer Patienten kennen und erfüllen würden, nicht mit einer tatsächlichen Entscheidung des Patienten gleichzusetzen, auch wenn in beiden Fällen die gleiche Entscheidung getroffen wurde [Barnhill und Miller, 2015]. Entsprechend ist die paternalistische Haltung, dass Fachpersonen im Gesundheitswesen für ihre Patienten entscheiden können und sollten, sowohl aus empirischen als auch aus ethischen Gründen abzulehnen.

In Bezug auf den Ansatz des vernünftigen Standards argumentieren Beauchamp und Childress [2009, S. 123], dass vor allem praktische Gründe gegen seine Umsetzung sprechen, da die Annahme eines hypothetischen vernünftigen Patienten zu abstrakt ist, um in der Praxis zu funktionieren. Außerdem ist die Anwendbarkeit dieses Standards tatsächlich davon abhängig, wie gut Behandler in der Lage sind, den «idealisierten rationalen Patienten» (wobei auch anzuzweifeln ist, dass dieser überhaupt existiert) zu imaginieren. Wir gehen aber davon aus, dass die grundlegende Annahme dass es empirisch ermittelbare und behandlungsspezifische Informationen gibt, die betreffende Patienten wahrscheinlich benötigen, damit sie eine informierte Entscheidung treffen können - durchaus vertretbar ist. Wir plädieren deswegen dafür, dass die Aufklärung über das Vorgehen beziehungsweise die dabei verwendete Heuristik bezüglich der Patienteninformation tatsächlich auch Bestandteil der betreffenden Patienteninformation sein sollte.

In einer solchen Patienteninformation kommt dem subjektiven Standard eine bedeutende Rolle zu. Beauchamp und Childress [2009, S. 124] gehen entsprechend davon aus, dass diese Heuristik «der präferierte moralische Standard für die Patienteninformation und informierte Einwilligung ist, da nur dieses Vorgehen das Informationsbedürfnis der betreffenden Person erfüllt». Gleichzeitig wird aber von den Autoren eingeräumt, dass dieser Standard in der klinischen Praxis kaum jemals vollständig umgesetzt werden kann, da man nicht davon ausgehen kann, dass ein Behandler immer eine ausrei- chend erschöpfende Analyse des Kontextes und der Persönlichkeit des Patienten durchführen kann, um so festzustellen, was im betreffenden Fall die notwendigen Informationen wären.

Wir gehen davon aus, dass eine Kombination des vernünftigen und des persönlichen Standards die Grundlage für Patienteninformation und informierte Einwilligung darstellen sollte. Aber es muss natürlich berücksichtigt werden, dass Patienten nicht immer in der Lage sind zu beurteilen, welche Informationen für die Entscheidungsfindung trivial und welche bedeutend sind [Blease, 2015c]. Beispielsweise konnte gezeigt werden, dass die Art des Krankheitsverständnisses bezüglich der eigenen depressiven Störung deren langfristigen Verlauf negativ beeinflussen kann [Blease, 2014]. Das Verständnis der eigenen Beschwerden hängt dabei auch von den Informationen ab, die der Patient von seinem Behandler erhält. Darüber hinaus zeigt sich auch, dass die Art der Informationsvermittlung einen erkennbaren Einfluss sowohl auf die Entscheidungsfindung als auch auf das Erleben der Beschwerden hat [Gigerenzer, 2007; Alfano, 2015; Blease, 2015c]. Entsprechend ist nicht nur der inhaltliche Aspekt der Patienteninformation für die Entscheidungsfindung des Patienten von Bedeutung, sondern auch die Art und Weise, wie diese Information vermittelt wird.

Ohne Anspruch auf Vollständigkeit erscheinen die folgenden Informationskategorien als sinnvolle Bestandteile einer hilfreichen Patienteninformation: Ergebnisse des diagnostischen Prozesses; Informationen über die vorliegende Störung; Informationen über Behandlungsmöglichkeiten, inklusive möglicher Alternativen; Vorgehen bei der Behandlung, d.h. Information über Ziele, zu erwartende Vorgehensweisen und den zu erwartenden Verlauf, zu erwartende Wirkungen, Nebenwirkungen und Risiken sowie die zu erwartende Dauer der Behandlung. Zusätzlich sollte dabei auch auf die Art und Weise der Vermittlung dieser Informationen geachtet werden, da diese einen erkennbaren Einfluss auf das Befinden von Patienten hat, sodass dieser Punkt bei der Beurteilung der Patienteninformation und der informierten Einwilligung entsprechend mit berücksichtigt werden muss.

\section{Genügt die Psychotherapie ihren eigenen ethischen Ansprüchen?}

Wird in der psychotherapeutischen Praxis - unter Berücksichtigung der vorangegangenen Erläuterungen - die Verpflichtung zur adäquaten Patienteninformation und informierten Einwilligung eingehalten? Auch wenn es schwierig ist, das Vorgehen bei der Patienteninformation in der klinischen Alltagspraxis zu ermitteln, gehen wir davon aus, dass die Autonomie von Patienten im oben genannten Sinne nicht vollumfänglich respektiert wird und dass Psychotherapeuten den eigenen ethischen Standard (unbeabsichtigt) nicht erfüllen. Unsere Vermutung dabei ist, dass es Psychotherapeuten versäumen, ihre Patienten über die Wirkungsweise von Psychotherapie grundsätzlich zu informieren.

Selbst wenn vereinzelt das grundlegende Konzept der evidenzbasierten Praxis abgelehnt wird [Tanenbaum, 2003], ist die Relevanz der Psychotherapieforschung für die klinische Praxis unbestritten 
[z.B. Wampold und Imel, 2015]. Es ist dennoch erstaunlich, dass es bislang noch zu wenig Diskussion darüber gibt, wie die Ergebnisse der Psychotherapieforschung an die Patienten weitergegeben werden. Und auch wenn die Ergebnisse der Psychotherapieforschung für Psychotherapeuten zunehmend handlungsleitend werden, besteht immer noch Uneinigkeit darüber, wie dieses Forschungsgebiet am besten konzeptualisiert werden sollte. Die APA definiert die evidenzbasierte Praxis als die Integration von wissenschaftlichen Forschungsergebnissen in die klinische Expertise im Zusammenhang mit den Eigenschaften, kulturellen Hintergründen und Präferenzen der Patienten [APA Presidential Task Force on Evidence-Based Practice, 2006]. Dieses Verständnis umfasst eine Vielzahl von verschiedenen wissenschaftlichen Erkenntnissen zu Ätiologie, Diagnostik und Behandlung aus der klinischen wie auch der psychologischen Forschung. Das alternative Konzept der «research-supported psychological treatments» (ehemals die «empirically supported treatments», EST) wird häufiger verwendet und ist auch schlanker; es bezieht sich auf das Verständnis der pharmakologischen Forschung und basiert primär auf dem Vergleich zwischen verschiedenen psychotherapeutischen Behandlungen [Wampold und Imel, 2015, S. 27; Goldfried, 2013]. Beispielsweise führen sowohl die BACP als auch die APA eine Liste von spezifischen Psychotherapien für bestimmte psychische Störungen, wohingegen Hinweise auf die relative Wirksamkeit und die allgemeinen Wirkmechanismen dieser verschiedenen Psychotherapien fehlen.

Es ist diese Auslegung von «Evidenz», die von Bedeutung ist. Beispielsweise gehen Vertreter des sogenannten Kontextmodells davon aus, dass der Einfluss von spezifischen Techniken für das Therapieergebnis irrelevant ist und nur zu 1\% der Varianzaufklärung beiträgt [Wampold, 1997; Wampold und Imel, 2015]. Diese Auffassung wird nicht von allen geteilt [z.B. Lambert und Barley, 2002], auch wenn die Ergebnisse auch von Kritikern des Kontextmodells bestätigt werden [Marcus et al., 2014]. Wir wollen uns aber in dieser Debatte nicht verlieren und vielmehr auf den Konsensus fokussieren, dass allgemeine Wirkfaktoren einen bedeutsamen Anteil an der Wirkung von Psychotherapie haben. Diese allgemeinen Wirkfaktoren beinhalten die therapeutische Beziehung, die gemeinsame Zielvereinbarung zwischen Patient und Psychotherapeut, Therapeutenfaktoren wie positive Wertschätzung, Empathie und Echtheit sowie Patientenfaktoren und -erwartungen. Auch wenn Uneinigkeit bei der Beurteilung des jeweiligen Stellenwerts dieser Faktoren innerhalb des Gesamteffekts von Psychotherapie besteht, ist es allgemein akzeptiert, dass diese Faktoren einen gewichtigen Anteil an den psychotherapeutisch erzielten Veränderungen haben [z.B. Beck, 1995; Lambert und Barley, 2002; Norcross, 2011; Wampold und Imel, 2015].

Mit Blick auf die fortwährende Debatte über empirische Evidenz zur Wirkung und Wirkungsweise der Psychotherapie sehen wir Verbesserungsbedarf bezüglich der Patienteninformationen in folgenden zwei Punkten.

Erstens besteht die Möglichkeit, dass Psychotherapeuten - unabhängig vom empirischen Status der verschiedenen psychotherapeutischen Vorgehensweisen - die Patienteninformation nicht angemessen an ihre spezifische Behandlung anpassen:
«Der Inhalt der Patienteninformation von einsichtsorientierten Psychotherapien kann sich substanziell von dem eher symptomfokussierter Behandlungen unterscheiden. Während Letztere, wie beispielsweise Expositionstherapien für spezifische Phobien, vor allem auf die Symptomreduktion abzielen, setzen einsichtsorientierte Therapien auf ein neues Verständnis der eigenen Person sowie auf den Umgang mit anderen. Entsprechend ähneln die Patienteninformationen in einer symptomfokussierten Psychotherapie eher denen in pharmakologischen Behandlungen, d.h. Ziele, Risiken und Vorgehensweisen sind konkret beschreibbar. In einsichtsorientierten Psychotherapien sind die Ziele, Vorgehensweisen und Prozesse hingegen eher komplex und wenig planbar, und Patienteninformationen müssen entsprechend offen formuliert werden» [Trachsel et al., 2015].

Beispielsweise zeigten die Ergebnisse einer US-amerikanischen Befragung, dass sich Patienteninformationen sowie der Umgang mit Vertraulichkeit, Risiken, Behandlungslänge, -vorgehen und -alternativen zwischen Vertretern verschiedener psychotherapeutischer Ansätze deutlich unterschieden [Somberg et al., 1993] und dass sich vor allem psychodynamische Psychotherapeuten skeptisch über die Möglichkeit und den Wert der Patienteninformation äußerten [Goddard et al., 2008]. Wir gehen davon aus, dass eine angemessene Information über die psychotherapeutische Vorgehensweise dieselbe entmystifiziert und in der Folge die Angst vor der Psychotherapie reduziert [Fisher und Oransky, 2008; Boswell et al., 2015].

Zweitens (und wahrscheinlich noch bedeutsamer und dringlicher) besteht die Notwendigkeit, Patienten über die sogenannten allgemeinen Wirkfaktoren aufzuklären. Mit Blick auf die allgemeine Anerkennung dieser Wirkkomponenten in Forschung und Praxis und unter Berücksichtigung der vorangegangenen Diskussion einer adäquaten Patienteninformation gehen wir davon aus, dass Patienten über die Bedeutung dieser Faktoren für die Psychotherapie angemessen und verständlich aufgeklärt werden sollen [Blease, 2015a,b; Gaab et al., 2015].

Zusammenfassend sollten Patienten folgende Informationen erhalten, damit ihre Autonomie angemessen respektiert wird [Blease, 2015b]: (i) eine Vereinbarung über Ziele und Vorgehensweisen als Grundlage einer erfolgreichen Behandlung; (ii) die Bedeutung der therapeutischen Beziehung als wichtige Voraussetzung für den Verlauf und die Wirkung der Behandlung; Patienten sollten sich u.a. unterstützt, ermutigt und verstanden fühlen; (iii) Patienten sollten sich bewusst sein, dass ihre eigenen Erwartungen und Haltungen bezüglich der Therapie grundlegend bedeutsam sind für den Erfolg der Behandlung.

Die Umsetzung dieser Punkte soll am Beispiel der kognitiven Verhaltenstherapie dargestellt werden:

«Es ist nicht ausreichend, den Patienten zu sagen, dass die kognitive Verhaltenstherapie über die kognitive Restrukturierung maladaptiver oder falscher Kognitionen wirkt. Eine angemessene Patientenaufklärung umfasst die Information, dass die kognitive Verhaltenstherapie auf der Theorie der kognitiven Restrukturierung basiert und dass wissenschaftliche Untersuchungen gezeigt haben, dass diese Vorgehensweise am besten bei den Patienten 
wirkt, die sich auf diese Theorie und die daraus abgeleiteten Vorgehensweisen einlassen» [Blease, 2015b].

Psychotherapeuten sollten entsprechend ihre Patienten darüber aufklären, dass, falls diese sich nicht auf das psychotherapeutische Vorgehen einlassen können, eine andere psychotherapeutische Ausrichtung möglicherweise sinnvoller ist. Zusätzlich erscheint es uns angebracht, die Bedeutung der Therapeutenvariablen sowie die der therapeutischen Beziehung beispielsweise wie folgt zu thematisieren: «Während der Behandlung sollten Sie sich von mir unterstützt und ermutigt fühlen, mit mir über Ihre Probleme zu reden. Wenn Sie mit der Behandlung, aus welchen Gründen auch immer, nicht zufrieden sind, können Sie das gerne mit mir diskutieren. Wir können dann versuchen, das zu klären und zu ändern, es kann aber auch sein, dass ein anderer Psychotherapeut oder eine andere Psychotherapie für Sie besser ist. Das bedeutet dann aber nicht, dass die Psychotherapie gescheitert ist und dass es nicht einen passenden Psychotherapeuten oder eine passende Psychotherapie für Sie gibt.»

Auch wenn ein solches Vorgehen auf den ersten Blick ungewohnt oder gar abschreckend erscheint, ist dies kein hinreichender Grund dafür, Patienten nicht angemessen über diese zentralen Aspekte aufzuklären. Darüber hinaus ist es auch sinnvoll, zusätzliche technische Möglichkeiten zur Patienteninformation zu nutzen. Beispielsweise können Patienteninformations- und Feedbacksysteme eingesetzt werden, die auf der Basis der Psychotherapieforschung mögliche Therapieverläufe in der Praxis verbessern und zudem negative Behandlungsergebnisse verhindern können [Lambert, 2007; siehe auch Lutz et al., 2011]. Es gibt auch keine Hinweise dafür, dass eine solche Patienteninformation die Wirkung von Psychotherapie beeinträchtigen würde. Vielmehr gehen wir davon aus, dass eine entsprechende Aufklärung von Patienten positive Effekte auf die Psychotherapie haben wird, auch wenn dies natürlich eine empirisch zu klärende Frage ist. Durch eine solche Patienteninformation wird unterstützt, dass Patienten nicht die Empfänger paternalistischer Behandlung, sondern autonome und mündige Vertreter ihrer eigenen Sache sind, die dadurch die Möglichkeit erhalten, die Prozesse und Voraussetzungen der eigenen psychotherapeutischen Veränderung zu verstehen.

\section{Schlussfolgerung}

Patienteninformation und informierte Einwilligung sind nicht nur moralische Verpflichtungen, sondern bieten auch Vorteile für die Patienten. Selbst wenn die Psychotherapieforschung viele wichtige Fragen noch nicht abschließend geklärt hat und zudem die In- terpretation der vorliegenden Antworten nicht einheitlich ist, sollte dies einer angemessenen Patienteninformation und informierten Einwilligung nicht im Wege stehen. Beide tragen zum gegenseitigen Vertrauen und zur therapeutischen Beziehung bei und sind damit die Grundlage eines der wichtigsten und wirksamsten psychotherapeutischen Wirkfaktors [Lambert und Barley, 2002]. Außerdem kann ein Beitrag zur Reduktion von ungünstigen Therapieverläufen und von Therapieabbrüchen geleistet werden, wenn Patienten auf diese Weise davor bewahrt werden, fehlende Fortschritte in der Psychotherapie einzig auf sich zu beziehen [Blease, 2015b]. Neben der Information des Patienten über die Wirkung und Wirkkomponenten von Psychotherapie sollte natürlich - wie auch in anderen Behandlungskontexten - über die möglichen Risiken und Nebenwirkungen der Psychotherapie informiert werden [Lilienfeld, 2007; Ladwig et al., 2014], auch wenn dies immer noch nicht das übliche Vorgehen in der Psychotherapie ist. Schließlich ist auch zu beachten, dass Patienteninformation und informierte Einwilligung den Patienten zum aktiven Mitgestalter der eigenen Behandlung machen und damit die Selbstverantwortung stärken [Fisher und Oransky, 2008, S. 576; Beahrs und Gutheil, 2001], was nach Erkenntnissen der Psychotherapieforschung wiederum deren Ergebnis verbessert [z.B., Pope und Vasquez, 2007; Lambert, 2007; Lutz et al., 2011].

Aufgrund juristischer sowie ethischer Verpflichtungen stehen Psychotherapeuten in der Verantwortung, ihre Patienten angemessen und umfassend zu informieren und aufzuklären. Aus unserer Sicht ist dies mit einigen wenigen und umsetzbaren Veränderungen in der klinischen Praxis möglich.

\section{Dank}

Die Autoren danken den Gutachtern sowie Prof. Dr. Jens Gaab und Prof. Dr. Winfried Rief für die hilfreichen Kommentare und Verbesserungsvorschläge zu einer früheren Version dieses Textes.

\section{Disclosure Statement}

Es liegen keine Interessenskonflikte vor.

\section{Literatur}

Alfano M: Placebo effects and informed consent. Am J Bioeth 2015;15:3-12.

American Medical Association (AMA): AMA Code of Medical Ethics. Opinion 8.08 - Informed Consent, 2006. www.ama-assn.org/ama/pub/physician-resources/medical-ethics/code-medical-ethics/opinion808.page? (Zugriff 10. December 2015)
APA Presidential Task Force on Evidence-Based Practice: Evidence-based practice in psychology. Am Psychol 2006;61:271-285.

American Psychological Association (APA): 10.01 Informed Consent to Therapy, Ethical Principles of Psychologists and Code of Conduct. Washington, DC., APA, 2010.
Übersetzt von Prof. Dr. Jens Gaab,

Dr. med Dr. phil. Manuel Trachsel,

Prof. Dr. Martin grosse Holtforth 
Beahrs JO, Gutheil TG: Informed consent in psychotherapy. Am J Psychiatry 2001;158:4-10.

Beck J: Cogntive Therapy: Basics and Beyond. New York, Guilford, 1995.

Blease C: The duty to be well-informed: the case of depression. J Med Ethics 2014;40:225-229.

Blease C: Informed consent, the placebo effect, and psychodynamic psychotherapy; in Schramme T (ed): New Perspectives on Medical Paternalism. Dordrecht, Springer, 2015a, pp 163-181.

Blease C: Talking more about talking cures: cognitive behavioural therapy and informed consent. J Med Ethics 2015b;41:750-755.

Blease C: Authorized concealment and authorized deception: Well-intended secrets are likely to induce nocebo effects. Am J Bioeth 2015c;15:23-25.

Beauchamp T, Childress J: Principles of Biomedical Ethics. Oxford, Oxford University Press, 2009.

Boswell F, Kraus D, Miller S, Lambert M: Implementing routine outcome monitoring in clinical practice: benefits, challenges, and solutions. Psychother Res 2015;25: 6-19.

British Association for Counselling and Psychotherapy (BACP): Ethics for counselling and psychotherapy; in BACP (ed): Ethical Framework for Good Practice in Counselling and Psychotherapy, Lutterworth, 2013. www.itsgoodtotalk.org.uk/assets/docs/BACP-EthicalFramework-for-Good-Practice-in-Counselling-andPsychotherapy_1276615182.pdf (Zugriff 10. December 2015).

British Association for Counselling and Psychotherapy (BACP): Research. Effectiveness of counselling. www. bacp.co.uk/research/resources (Zugriff 10. December 2015).

Castonguay LG, grosse Holtforth M: Change in psychotherapy: a plea for no more 'nonspecific' and false dichotomies. Clin Psychol - Sci Pr 2005;12;198-201.

Di Blasi Z, Harkness E: Influence of context effects on health outcomes: a systematic review. Lancet 2001;357: 757-762.

Dsubanko-Obermayr K, Baumann U: Informed consent in psychotherapy: demands and reality. Psychother Res 2010;8:231-247.

Dworkin G: Paternalism; in: Zalta EN (ed): The Stanford Encyclopedia of Philosophy, summer ed. 2010. http:// plato.stanford.edu/entries/paternalism/ (Zugriff 10. December 2015).

Engelhardt Tristram $\mathrm{H}$ Jr: Advance directives and the right to be left alone; in Hackler C, Moseley R, Vawter DE (eds): Advance Directives in Medicine. New York, Praeger, 1989, pp 141-154.

Feinberg J: Legal paternalism. Canadian Journal of Philosophy 1971;1:105-124.

Fisher CB, Oransky M: Informed consent to psychotherapy and the American Psychological Association's Ethics Code. National Register of Health Service Psychologists, 2008a. www.nationalregister.org/pub/the-national-register-report-pub/the-register-report-spring-2008/ informed-consent-to-psychotherapy-and-the-americanpsychological-associations-ethics-code (Zugriff 10. December 2015).

Fisher CB, Oransky M: Informed consent to psychotherapy: protecting the dignity and respecting the autonomy of patients. Journal of Clinical Psychology 2008b;64: 576-588.
Frank JD, Frank JB: Persuasion and Healing. Baltimore, John Hopkins University Press, 1991.

Gaab J, Blease C, Locher C, Gerger H: Go open: a plea for transparency in psychotherapy. Psychol Conscious (Wash D C) 2015;DOI dx.doi.org.1037/cns0000063.

General Medical Council (GMC): Good Medical Practice, paragraph 36, 2010. www.gmc-uk.org/Good_Medical_ Practice__Archived.pdf_51772200.pdf (Zugriff 10. December 2015).

Gigerenzer G: Gut Feelings: Short Cuts to Better Decision Making. London, Penguin, 2007.

Goddard A, Murray CD, Simpson J: Informed consent and psychotherapy: an interpretative phenomenological analysis of therapists' views. Psychol Psychother 2008; 81:177-191.

Goldfried M: What should we expect from psychotherapy? Clin Psychol Rev 2013;33:654-662.

Greenwood J: Placebo control treatments and the evaluation of psychotherapy: a reply to Grünbaum and Erwin. Philos Sci 1997;62:605-621.

Grünbaum A: The placebo concept. Behav Res Ther 1981; 19:157-167.

Grünbaum A: The placebo concept in medicine and psychiatry. Psychol Med 1986;16:19-38.

Howick J: The Philosophy of Evidence-based Medicine. Oxford, Wiley-Blackwell, 2011.

Jensen KB, Kaptchuk TJ, Kirsch I, et al.: Nonconscious activation of placebo and nocebo pain responses. Proc Natl Acad Sci USA 2012;109:15959-15964.

Jopling D: Talking Cures and Placebo Effects. Oxford, Oxford University Press, 2008.

Kaptchuk TJ, Goldman P, Stone DA, et al.: Do medical devices have enhanced placebo effects? J Clin Epidemiol 2000;53:786-792.

Kaptchuk TJ, Kelley JM, Conboy LA, et al.: Components of placebo effect: randomized controlled trial in patients with irritable bowel syndrome. BMJ 2008;336:999-1003.

Kirsch I: Placebo psychotherapy: synonym or oxymoron? J Clin Psychol 2005;67:791-803.

Kirsch I, Wampold B, Kelley J: Controlling for the placebo effect in psychotherapy: noble quest or tilting at windmills? Psychology of Consciousness 2015;DOI dx.doi. org/10.1037/cns0000065

Ladwig I, Rief W, Nestoriuc Y: What are the risks and side effects of psychotherapy? - Development of an inventory for the assessment of negative effects of psychotherapy (INEP). Verhaltenstherapie 2014;24:252-264.

Lambert MJ: What we have learned from a decade of research aimed at improving psychotherapy outcome in routine care. Psychother Res 2007;17:1-14.

Lambert MJ: Bergin and Garfield's Handbook of Psychotherapy and Behavior Change, ed 6. San Francisco, Wiley, 2013.

Lambert MJ, Barley D: Research summary on the therapeutic relationship and psychotherapy outcome: expectations and preferences; in Norcross JC (ed): Psychotherapy Relationships That Work: Therapist Contributions and Responsiveness to Patients. London, Oxford University Press, 2002, pp 17-32.

Lilienfeld S: Psychological treatments that cause harm. Perspect Psychol Sci 2007;2:53-70.

Lutz W, Boehnke JR, Koeck K: Lending an ear to feedback systems: evaluation of recovery and non-response in psychotherapy in a German outpatient setting. Community Ment Health J 2011;47:311-317.
Marcus D, O'Connell D, Norris A, Sawaqdeh A: Is the Dodo bird endangered in the 21 st century? A metaanalysis of treatment comparison studies. Clin Psychol Rev 2014;34:519-530.

Norcross JC: Psychotherapy Relationships That Work: Evidence-based Responsiveness. New York, Oxford University Press, 2011.

Pope KS, Vasquez MJ: Ethics in Psychotherapy and Counseling: A Practical Guide. San Francisco, Wiley, 2007.

Rosenthal D, Frank J: Psychotherapy and the placebo effect. Psychol Bull 1956;55:294.

Rosenzweig S: Some implicit common factors in diverse methods of psychotherapy. Am J Orthopsychiat 1936; 6:412-415.

Shapiro AK, Shapiro E: The Powerful Placebo: From Ancient Priest to Modern Physician. Baltimore, John Hopkins University Press, 1997.

Silber TJ: Treatment of anorexia nervosa against the patient's will: ethical considerations. Adolesc Med State Art Rev 2011;22:283-288.

Somberg DR, Stone GL, Claiborn CD: Informed consent: therapists' beliefs and practices. Prof Psychol Res Pr 1993;24:153-159.

Street R, Haidet P: How well do doctors know their patients? Factors affecting physician understanding of patients' health beliefs. J Gen Intern Med 2011;26:2127.

Tanenbaum SJ: Evidence-based practice in mental health: practical weaknesses meet political strengths. J Eval Clin Pract 2003;9:287-301.

Topol E: The Patient Will See You Now: The Future of Medicine in Your Hands. New York, Basic Books, 2015.

Trachsel M, grosse Holtforth M, Biller-Andorno N, Appelbaum PS: Informed consent for psychotherapy: still not routine. Lancet Psychiatry 2015;2:775-777.

Trachsel M, Mitchell C, Biller-Andorno N: Advance directives between respect for patient autonomy and paternalism; in Lack P, Biller-Andorno N, Brauer S (eds): Advance Directives, International Library of Ethics, Law, and the New Medicine 54. Dordrecht, Springer, 2013, pp 169-179.

Turner A: 'Placebos' and the logic of placebo comparison. Biol Philos 2012;27:419-432.

UK Department for Constitutional Affairs: Mental Capacity Act 2005: Code of Ethics. www.gov.uk/government/ uploads/system/uploads/attachment_data/file/224660/ Mental_Capacity_Act_code_of_practice.pdf (accessed December 16, 2015).

Veatch R: Patient, Heal Thyself: How the New Medicine Puts the Patient in Charge. Oxford, Oxford University Press, 2009.

Wampold BE, Imel ZE: The Great Psychotherapy Debate: Models, Methods, and Findings, ed 2. New York, Routledge, 2015.

Wampold BE, Mondin GW, Moody M, et al.: A meta-analysis of outcome studies comparing bona fide psychotherapies: empirically, 'all must have prizes'. Psychol Bull 1997;122, 203-215. 